\title{
Use of mechanical refining to improve the production of low-cost sugars from lignocellulosic biomass
}

\author{
Junyeong Park ${ }^{1}$, Brandon Jones ${ }^{1}$, Bonwook $\mathrm{Koo}^{2}$, Xiaowen Chen ${ }^{3}$, \\ Melvin Tucker ${ }^{3}$, Ju-Hyun $\mathrm{Yu}^{4}$, Thomas Pschorn ${ }^{5}$, Richard Venditti ${ }^{1}$, Sunkyu Park ${ }^{1,6^{*}}$ \\ ${ }^{1}$ Department of Forest Biomaterials, North Carolina State University, Raleigh, NC 27695, USA \\ ${ }^{2}$ SK Innovation, Daejeon, Republic of Korea \\ ${ }^{3}$ National Bioenergy Center, National Renewable Energy Laboratory, Golden, CO 80127, USA \\ ${ }^{4}$ Korea Research Institute of Chemical Technology, Daejeon, Republic of Korea \\ ${ }^{5}$ Andritz, Montréal, QC, H8T 3H4, Canada \\ ${ }^{6}$ Department of Forest Sciences, Seoul National University, Seoul, Republic of Korea \\ * Corresponding author: sunkyu_park@ncsu.edu
}

\begin{abstract}
Mechanical refining is widely used in the pulp and paper industry to enhance the end-use properties of products by creating external fibrillation and internal delamination. This technology can be directly applied to biochemical conversion processes. By implementing mechanical refining technology, biomass recalcitrance to enzyme hydrolysis can be overcome and carbohydrate conversion can be enhanced with commercially attractive levels of enzymes. In addition, chemical and thermal pretreatment severity can be reduced to achieve the same level of carbohydrate conversion, which reduces pretreatment cost and results in lower concentrations of inhibitors. Refining is versatile and a commercially proven technology that can be operated at process flows of $\sim 1,500$ dry tons per day of biomass. This paper reviews the utilization of mechanical refining in the pulp and paper industry and summarizes the recent development in applications for biochemical conversion, which potentially make an overall biorefinery process more economically viable.
\end{abstract}

\section{Introduction}

A bioeconomy offers a low carbon emission solution to the ever increasing petroleumbased chemical and fuel demands, climate change, and energy security. However, these benefits cannot be realized without significant improvements in biorefinery technologies. One of the greatest challenges is the ability to access low cost sugars and generate products that are costcompetitive with products derived from petroleum and natural gas. In the biochemical conversion pathway, the cost of overcoming biomass recalcitrance through chemical and enzymatic treatment adds significant cost to the overall biorefinery production process. 
Recently, mechanical refining has been proposed as a viable process that can overcome biomass recalcitrance by opening up the biomass structure, which is different from particle size reduction - another category of mechanical treatment on biomass. In particle size reduction, biomass undergoes significant size reduction by mechanical force before or after pretreatment process. However, this kind of intensive structural changes requires high energy consumption (Zhu et al., 2010). The alteration of biomass using mechanical refining is more focused on opening up complex and strongly-integrated biomass structure by external fibrillation and internal delamination, compared to increasing of surface area by reducing particle size of biomass, which will be covered in more detail in this review.

Refining is an established technology in the pulp and paper industry, which can enhance the end-use properties of paper products. The purpose of refining in the pulp and paper industry is to separate fiber bundles and to create high surface area by fibrillation of cell wall structures. This allows fibers to be easily collapsed to a ribbon-like shape upon press and drying operation and bonded together with adjacent fibers to produce a uniform and strong fiber network. This structural and morphological alteration of fibers by mechanical refining can also be beneficial for biomass conversion processes. There has been several studies about the effect of mechanical refining on subsequent enzymatic saccharification (Chen et al., 2013; Chen et al., 2014; Jones et al., 2014; Jones et al., 2013; Zhu et al., 2009a; Zhu et al., 2009b). Despite being identified for its potential in combination with chemical pretreatment, the use of mechanical refining in biochemical conversion processes is still in initial stage. There are still many opportunities for improvement of this process to facilitate the production of low cost sugars from recalcitrant lignocellulosic biomass.

In this review, the current utilization of mechanical refining in the pulp and paper industry and how it can be applied to biochemical conversion process will be covered. The recent development of this technology for sugar production and its optimization opportunity will be addressed in detail.

\section{Mechanical refining in the pulp and paper industry}

There are many different paper products and each of them requires specific performance properties to meet their end-use application standards. Apart from some specific factors, there is one basic requirement for most paper products - evenly distribution and strong bonded cellulose fibers. This can be achieved by a well-controlled mechanical treatment operation on the fibers. For the utilization of cellulose fibers in paper products, the pulping process is necessary to extract individual wood fibers. The pulping process can be divided into two categories, chemical pulping and mechanical pulping. The following description will be focused on the use of mechanical refining in the most common chemical pulping process (i.e. Kraft pulping), especially for high Kappa pulp (high lignin content) production. Chemical pulping uses digestion of wood chips with heat and chemicals to break down and separate the lignin, which binds the cellulose fibers together in the native biomass, without substantial loss of the fiber itself. There 
are several different types of mechanical refining applications during the pulping and papermaking processes (Smook, 2002).

Briefly, after high Kappa cooks of wood chips, some fibers will not be fully separated. A blowline refiner is generally used at the end of chemical pulping to fiberize cooked chips. Even after blowline refining, there can still be shives (small fiber bundles) left in the pulp. De-shive refining is commonly used to reduce the amount of shives and disintegrate the fibers for downstream processing. During papermaking, stock preparation refining is used to develop external fibrillation and increase the flexibility of the cell wall. To make a strong product, cellulose fibers need to make an entangled network attached to one another by hydrogen bonding. The increased number of hydrogen bonds at a crossing point between fibers will increase the strength of the linkage. The swellability of the fiber can also impact the relative bonded area between fibers. This action can be promoted through the loosening of microfibrils from the compact bundle of fibril strands within and external to the fiber wall.

\subsection{Types of mechanical refiners in the pulp and paper industry}

There have been several different types of refiners utilized in the history of the pulp and paper industry, but most modern mills are adopting disc refiners. Disc refiners are a more recent development and basically consist of three components: a rotating disc plate that is connected to the motor, a stationary disc plate, and housing (Figure S1). These disc plates are enclosed within a solid housing and can operate at atmospheric pressure or as a pressurized vessel. Two different inlet designs are available, an inlet pipe system for low consistency slurries or a screw/conveyor feeding system for high consistency material (usually higher than 10\%). Disc refiners offer many advantages over older-type refiners (e.g. Hollander or valley beater, conical refiner, etc.) including lower no-load energy consumption, applications with higher stock consistency and higher biomass loading, versatility in refiner plate design, and lower capital cost (Smook, 2002).

There are several different purposes of the refining process, which determine where refiners need to be installed within the process layout. Generally, the purpose of mechanical refining in the process can be divided into three categories: (1) fiberization of cooked wood chips after digester, (2) reduction and disintegration of large particles or fiber bundles (shive), and (3) surface fibrillation and internal delamination of cell wall to improve paper properties. For high Kappa pulp production (high lignin content), all three types of refiners are commonly used, while the last one is only used for low Kappa pulp production (low lignin content).

The first one is widely referred to as blowline refining, which is used to fiberize cooked chips. If the feed is directly connected with a continuous digester, the operation of this type of refiner is done under high pressure. In case of a batch-type digester, cooked chips are collected in a blow tank, then fed into a refiner for fiberization. This refiner is called a hot stock refiner and operates under atmospheric condition. The main purpose of this refining process is to separate individual fibers from bundles. If this refining step is too severe, it can generate fibrillated fibers and fines, which will affect the drainability of pulp. Because low dewatering for cooked and 
fiberized pulp can create an issue during subsequent washing processes, blowline refining is optimized within the acceptable range of drainability.

The second type, a de-shive refiner, can be applied afterward to disintegrate fiber bundles and reduce the shive content. A shive can be defined as a bundle of incompletely separated fibers, which can be shown as dirt in paper or board. It can cause cosmetic defects in the paper products, as well as some performance problems, including low print quality and low tensile and burst strength. A de-shive refiner operates under atmospheric condition, following the washing step of cooked and fiberized fibers with a medium or high consistency loading.

The third type of mechanical refining is stock preparation refining. During this mechanical treatment process, individual fibers become more easily-collapsible with a large amount of exposed bonding sites. The fiber structure from the wood can be visualized as a cylindrical shape with longitudinal void inside, called the lumen. The native wood cells are connected by the middle lamella, which has a high lignin concentration, compared to the inner part of fiber cell wall (e.g., S2 layer). The outer fraction of the fiber cell wall is the primary wall (P), followed by secondary (S1, S2, S3) wall layers, which are characterized by distinct fibril alignments, making up the bulk of the cell wall.

To make the fiber flexible, collapsible, and have more accessible bonding sites, it is necessary to disrupt the well-oriented structure of the cell wall layers and to let the S2 layer, which accounts for most of the carbohydrates in the biomass, become swollen with water. Stock preparation refining can induce these changes in the fibers. In the case of a conventional disc refiner, for example, the stock flow is fed into the rotating refining discs, which have complex designs with bars and grooves, and pulp fibers undergo mechanical processing (Figure S2a). Once fiber bundles are picked up between disc bars, refining action damages and removes $\mathrm{P}$ and S1 cell wall layers, exposing the S2 layer to water. After the S2 layer is exposed and swollen, mechanical action causes the layer to delaminate and thus the fibers are collapsed. The mechanical action required for proper refining is not a simple brushing, cutting, grinding or pounding. Rather, it is a more precise shearing of the fibers between two plate surfaces, usually rotating and stationary discs with protrusions. The cyclic shearing, compression, and release of fibers between moving surfaces induces the desired effects of refining such as external fibrillation, internal delamination and fiber cutting. Overall, the principal objectives of stock preparation refining are (a) to increase the flexibility of the cell wall to make more fiber-to-fiber contact area upon papermaking and (b) to fibrillate the external surface of the fiber to increase the total available bonding sites.

As mentioned previously, there is another pulping method using mainly a mechanical process. In mechanical pulping, a machine is used to grind or refine wood chips to make fibers and fiber fragments. This process requires a substantially higher amount of energy to separate fibers than chemical pulping, and there is little or no removal of cell wall components during the process. Most modern mechanical pulping mills use large disc refiners, but the process of mechanical pulping is different from the stock preparation refining of fibers. 


\subsection{Mechanical refining effects on fiber structure}

Mechanical stress on cellulose fibers during the refining process can induce different alterations to the fiber structure and morphology (Figure S2b). The effects can be classified into three modes: external fibrillation, internal delamination, and cutting.

External fibrillation: During refining, flocs of fibers undergo several types of stress, such as compression and shear stress. The impact of refiner bars, as well as bar-fiber and fiber-fiber friction from mechanical stress, will induce fracture of the fiber structure and peeling of fibrils from the fiber surface. When fibrillated components are still attached to the fiber surface, this alteration is referred to as external fibrillation. It is considered a major desirable effect of refining, due to the concept that external fibrils serve as bonding agents for the inter-fiber network. External fibrillation of lab-scale refined fiber has been investigated using light microscopy and electron microscopy (Kibblewhite, 1972; Molin and Lennholm, 2000; Szwarcsztajn and Przybysz, 1972; Teder, 1964). The degree of fibrillation can be quantified by measuring the increase in the specific surface of the long fiber fraction (Page, 1989).

Internal delamination: Mechanical stress during the refining process affects not only the structure of the external surface on fiber, but also the internal structure of cell wall. The refining of chemical pulp increases fiber flexibility and the changes in the internal structure of the fiber are considered to be responsible for this property. Mechanical stress will break the crosslinking of interfibrillar matrices, resulting in loosened internal structure and consequent swelling of fibers (Page, 1989). Internal delamination generates cavities within the cell wall structure, through which water can flow into the internal structure by capillary forces. This causes swelling, which is often used as an indicator of the degree of refining. Internal delamination induced by the refining process has also been observed by light and electron microscopic methods in studies (Brändström et al., 2005; Molin and Daniel, 2004; Page, 1989).

Fiber cutting (shortening): Fines, which are defined as loose fibrous material that are less than $0.3 \mathrm{~mm}$, are inevitably produced during mechanical refining as a result of fiber shortening or removal of fibrils from fibrillated and delaminated cell walls. Generated fines consist mostly of fragments of the P and S1 layers of the fiber cell wall due to the abrasion of fibers against each other or against refiner bars. The decrease in fiber length is generally undesirable. This is because fines are difficult to retain in the product and can cause a significant decrease in drainage rate which results in slower operation speeds. In addition, fines can decrease paper properties such as formation and strength (Stoere et al., 2001), while it can be a desired effect to improve formation by decreasing a crowding number in some rare applications (Kerekes, 1990; Stoere et al., 2001).

\subsection{Mechanical refining variables}

There are several refiner plate and operational variables that can greatly affect the refining process parameters such as the number of impacts and residence time of fibers in the refining zone (Kure and Dahlqvist, 1998; Kure et al., 2000). To produce fibers with optimal properties in final products at minimum energy consumption, it is important to understand and 
optimize these variables. For example, refiner plate design can be adjusted to meet specific pulp quality requirements by manipulating factors such as bar width and angle, groove width and depth, number of refining zones, inlet geometry, and metallurgy. Some of these variables are summarized in this section.

Bar Width: In the absence of potential metal contamination and no-load power concerns, the width of bars would be only required to rigidly hold the flocs of pulp that are being deformed. In real situations, the bar width is dictated mostly by the metal contamination potential of the application, which can introduce bending loads on the bars that far exceed the normal refining load. Similar to the refiner plate bar width is a plate property known as the 'bar edge length' (BEL), Sometimes referred to as the 'cutting edge length', the BEL is a measurement of the total length of bar edges that will impact the fibers during one plate revolution $(\mathrm{km} / \mathrm{rev})$. All other parameters being constant, changing the plate design to finer periphery bars will increase the BEL $(\mathrm{km} / \mathrm{rev})$ and correspondingly reduce the overall refining intensity.

Groove Width: The minimum practical groove width is usually determined by the tendency for plugging of the groove, either by fiber or a common contaminant. Groove width is dependent on the average fiber length of the species being refined. Another factor to consider is that no-load power varies inversely with the hydraulic section or open area of the cross section of the pattern. A plate with narrow grooves and wide bars will have a higher no-load power than a plate with wide grooves and narrow bars. Minimum bar and groove widths create the lower limit of refining intensity for any given refiner size operating at a fixed speed. If there is a strong quality incentive to reduce intensity further, it can only be done by adding additional equipment. Conversely, refining intensity can be increased by increasing the bar and groove widths. In general, high intensity refining yields more fiber cutting and low intensity refining achieves more fibrillation. The mathematical explanations and definitions of refining intensity are described in section 2.4.

Gap size: The clearance, also known as plate gap, between the refiner discs affects the energy applied to fibers. The adjustment of plate gap is usually accomplished by regulating the hydraulic pressure applied to the stator disc. In some refiners, an electromechanical gear motor is applied to control the gap width. A narrower gap results in more compressed fibers between the discs, which can cause higher friction generated between the plates and increased energy load. Widening the gap can reduce the refining intensity, while also reducing the relative refining energy load. Often, for commercial applications, the plate gap width is varied within a feedback control loop to create a constant refining intensity.

Residence time: The time during which the fibers are subjected to mechanical stress between refiner discs is referred to as the residence time. The residence time of fibers in the refining zone can be controlled by changing flow rate, plate design (Høydahl et al., 1995; Kure et al., 2000) and adjusting the speed of refiner discs (Kure et al., 2000). In a pressurized disc refiner, the residence time can be increased by increasing the pressure differential between the outlet and inlet streams. The changes of these two operation factors will affect not only the residence time, but the number of impacts on fibers, resulting in a change of refining intensity. Hence, the 
residence time will determine specific energy per plate bar impact and energy applied on the unit amount of refined fibers.

Disc rotation speed: Increasing refiner disc speed is related to the refining intensity. It has been reported that refining intensity is proportional to the square of disc rotating speed (Miles, 1991). Sabourin et al. presented that at the same level of refining intensity, the rate of energy transfer (specific refining energy) was three to four times higher when the refining intensity was increased by disc speed, compared to increased intensity by plate design in pulping process (Sabourin et al., 1997). Higher refining speed yielded more fibers with broken circumference (longitudinal splits), but there was little changes in fiber length after refining (Kure et al., 1999). Hence, refining with high disc speed can reduce the energy consumption required per unit amount of pulp, while retaining the same level of refining intensity.

\subsection{Energy consumption}

The amount of energy input during mechanical refining is an important factor in determining economic viability, as well as the main parameter to estimate the amount of work done on the fibers. The energy transferred to the pulp is considered as specific energy, and each fiber type has an optimal level of specific energy for maximum fiber development by refining. The 'net specific energy' (NSE) can be calculated as the following equation:

$$
\operatorname{NSE},\left(\frac{k W h}{t}\right)=\frac{\text { Net load }}{\text { Dry mass of fibers per hour }}=\frac{\text { Refining load }- \text { No load }}{\text { Dry mass of fibers per hour }}
$$

Where the mass of fibers can be calculated by multiplying the consistency of pulp by the mass flow rate of pulp into the refiner. No load energy is usually 10 20\% of total energy load in commercial refining systems. The applied energy is dependent on two parameters - refining intensity and number of bar impacts. Refining intensity is controlled by motor load during the process and the load is a response variable to the width of refiner plate gap. This is a common term used in refining to describe how the energy is applied to the pulp by determining the amount of energy transferred to the pulp in one second across one meter of a refiner plate's bar edge. The 'refining intensity' (I), also known as 'specific edge load' (SEL), can be calculated based on the applied power divided by the product of rotating speed and bar edge length:

$$
\text { SEL or } I,\left(\frac{W s}{m}\right)=\frac{\text { Refining load }- \text { No load }}{\text { rpm } \times \text { Bar edge length } \times \min / 60 s}
$$

Where the bar edge length is a property of the specific plate design expressed in $\mathrm{km} / \mathrm{rev}$ unit, which is the total length of bar edges that the fibers will see in one revolution. Additionally, a parameter independent of energy for refining can indicate the average 'number of deformations' $(\mathrm{N})$, or the impact length per unit mass, can be calculated by the following equation: 


$$
N,\left(\frac{\mathrm{km}}{\text { ton }}\right)=\frac{\text { rpm } \times \text { Bar edge length } \times 60 \mathrm{~min} / 1 \mathrm{hr}}{\text { Dry mass of fibers per hour }}
$$

The number of bar impacts during refining is closely related to the flow rate of the pulp through the refiner. This calculation of $\mathrm{N}$ assumes that the deformation at any refiner bar crossing point occurs over a finite time interval and that the average deformations per unit time is directly proportional to the rotational speed. Again, plate design and rotational speed are also process variables affecting the number of bar impacts on the pulp. Then, the amount of refining energy is equal to the magnitude of the refining intensity (I) multiplied by the number of deformations per unit mass $(\mathrm{N})$. Plate design, consistency of pulp, and rotational speed of the motor are all process variables that affect the amount of refining energy applied to the pulp.

The typical specific energy input for different grades of paper products is presented in Table 1. There are several different parameters that affect the energy consumption during refining and their relationship with generated fiber characteristics is a complex inter-related function. Therefore, it is necessary to find the optimal energy input based on the type of fiber and its end-use applications. In general, low intensity refining is more beneficial for most all papermaking applications because it can improve product strength properties and reduce the energy requirement. This reduction in refining intensity can be achieved by increasing the refining speed (rpm) or by changing to a finer (higher $\mathrm{km} / \mathrm{rev}$ ) bar edge length plate design. Common refining intensity ranges for different paper grades are shown in Table 1.

\section{Mechanical refining in biochemical conversion}

Lignocellulosic biomass has intrinsic characteristics that are recalcitrant to enzymatic hydrolysis to produce sugars, which is a major challenge in the commercialization of cellulosebased biofuels and chemicals (Himmel et al., 2007). One of the most recent developments in the biochemical conversion of lignocellulosic biomass is the inclusion of mechanical refining, common to the pulp and paper industry. As described in the previous section, mechanical refining systems have been widely used to increase the specific surface area of wood fibers through the combination of targeted fibrillation and delamination of cellulose fibers. The same technology can be directly applied to biomass deconstruction to increase enzyme accessibility and the subsequent carbohydrate conversion efficiency (Chen et al., 2012b; Ertas et al., 2014; Huo et al., 2013; Jones et al., 2013; Ju et al., 2013; Xu et al., 2014; Zhu et al., 2009b).

By implementing mechanical refining technology, biomass recalcitrance and process complexity can be significantly reduced for the following reasons. a) Carbohydrate recovery can be enhanced with commercially attractive levels of enzymes, and enzyme dosages can be reduced since the substrate is more readily digestible. b) Pretreatment severity (e.g. dilute-acid treatment) can be greatly reduced to achieve the same level of sugar recovery, which reduces the pretreatment cost and results in lower concentrations of inhibitors (e.g. carbohydrate degradation products) that allows fermentation or catalytic upgrading to be more effective. c) Mechanical refining technology is versatile and can be installed in conjunction with any type of process 
scheme including autohydrolysis, dilute-acid, and alkaline pretreatment. d) Mechanical refining is a commercially proven technology at process flows of $\sim 1,500$ dry tons per day of biomass.

\subsection{Improvement in carbohydrate conversion at reduced enzyme dosage}

One of the major advantages of mechanical refining is the ability to achieve high carbohydrate conversion with lower amounts of enzyme loading. With dilute-acid pretreated corn stover presented in Figure 1a, the carbohydrate conversion yield without refining at 4 FPU/g of enzyme loading was $78 \%$, which is $9 \%$ higher than the conversion at 2 FPU/g. When the pretreated biomass was refined with $2 \mathrm{k}$ revolutions in a lab-scale PFI refiner, the same conversion was achieved at $2 \mathrm{FPU} / \mathrm{g}$. This clearly demonstrates a reduction in enzyme dosage by half at the same level of conversion by utilizing mechanical refining. Moreover, after $4 \mathrm{k}$ revolutions PFI refining, there was little difference between 2 and 4 FPU/g of enzyme loading.

The advantage of mechanical refining to reduce the amount of enzyme loading has been reported in several studies. Mechanical refining treatment on green-liquor $\left(\mathrm{GL}, \mathrm{Na}_{2} \mathrm{CO}_{3}\right.$ and $\mathrm{Na}_{2} \mathrm{~S}$ ) pretreated hardwood improved the enzymatic saccharification, where the carbohydrate conversion for the unrefined sample with $10 \mathrm{FPU} / \mathrm{g}$ enzyme loading was substantially enhanced from $51.5 \%$ to over $60 \%$ even with half of the enzyme loading (5 FPU/g) after mechanical treatment by PFI refining with $8 \mathrm{k}$ revolutions. With the same level of enzyme loading (10 FPU/g), the refined sample achieved $70 \%$ of carbohydrate conversion. It was explained that this might be attributed to the enhancement in enzyme accessibility on pretreated biomass, which was supported by the measurement of increased surface area and pore volume with mechanical refining (Koo et al., 2011). With low severity dilute-acid pretreated corn stover, it was observed that the glucan conversion of the PFI-refined biomass with 50\% reduction in enzyme loading was similar to, or even higher than that of the unrefined biomass with full enzyme charge, depending on the varieties of feedstock (Chen et al., 2012b). The effect of refining was investigated on enzymatic hydrolysis for GL-pretreated hardwood and the carbohydrate conversion was increased from $67 \%$ to $90 \%$ after refining. Additionally, it was possible to reduce the enzyme loading by $32 \%$ after refining to achieve the target conversion yield of $91 \%$ (Jones et al., 2013). Drying of biomass can increase the recalcitrance of the biomass by irreversible pore closure (Park et al., 2006), termed hornification that can be reversed to some extent by mechanical refining. Chen et al. tested the effect of mechanical refining on wood pulps exposed to different drying methods and observed that refining enhanced the carbohydrate conversion, especially for hydrolysis conditions with lower enzyme loading (Chen et al., 2012a). Different types and scales of mechanical refining were compared with sodium carbonate $\left(\mathrm{Na}_{2} \mathrm{CO}_{3}\right)$ pretreated hardwood using a lab-scale PFI refiner, 12" disc pilot scale refiner, and 42" disc industrial scale refiner. It was shown that the 12 " and 42 " disc refining significantly increased the carbohydrate conversion to $65 \sim 70 \%$, compared to the unrefined biomass (36\%) and PFIrefined biomass (53\%) (Jones et al., 2014).

Mechanical refining can also increase the digestibility of softwood, which is considered to be the most recalcitrant biomass. The carbohydrate conversion data for the unrefined GL- 
pretreated biomass in Figure 1b showed only about 30\% of the theoretical carbohydrate conversion after $144 \mathrm{~h}$ of enzymatic hydrolysis incubation time. With the low-severity mechanical refining of $2 \mathrm{k}$ PFI refining, the conversion was substantially increased, resulting in up to $45 \%$ of carbohydrate conversion after $144 \mathrm{~h}$. With $6 \mathrm{k}$ PFI refining, the final conversion of $2 \mathrm{k}$-refined sample $(\sim 45 \%)$ was obtained at about half of the incubation time $(72 \mathrm{~h})$. This trend in increasing carbohydrate conversion by the addition of mechanical refining was consistent up to $8 \mathrm{k}$ revolution of PFI refining, with the ultimate conversion of over $60 \%$. Excessive refining, however, showed a slight decrease in the improvement of total carbohydrate conversion compared to the 8k PFI refined condition (10k revolution). It was reported that PFI refining on pretreated biomass increased the carbohydrate conversion, but beyond a certain degree of refining, the improvement in yields were marginal (Chen et al., 2013; Koo et al., 2011).

Zhu et al. showed that mechanical refining after sulfite pretreatment of two softwood species had increased glucose conversion yields to over 90\% (Zhu et al., 2009a). Wu et al. introduced both oxygen delignification and mechanical refining on GL-pretreated softwood and reported that, compared to the yield from the biomass with only GL-pretreatment, the two additional post-pretreatment processes increased the carbohydrate conversion by $14 \%$ and $19 \%$, respectively ( $\mathrm{Wu}$ et al., 2010). The combination of delignification and refining achieved a carbohydrate conversion of $78 \%$, which was $37 \%$ higher than that from the unrefined softwood with only the green-liquor pretreatment. Alongside the publications referred to above, there are more studies with different feedstocks, pretreatments, and refining methods, which have all shown the positive effect of mechanical refining on the biomass digestibility (Franco et al., 2012; Gil et al., 2009; Han et al., 2015; Hoeger et al., 2013).

\subsection{Variations in mechanical refining reactivity}

The effect of mechanical refining has interactions with enzyme loading and the pretreatment severity. Hydrolysis data was analyzed by plotting the absolute improvement in carbohydrate conversion after refining (difference in conversion between before and after) versus the carbohydrate conversion of the corresponding unrefined biomass at the same hydrolysis condition (Jones et al., 2013). As shown in Figure 2, there exists a maximum improvement point in terms of refining reactivity. When the pretreated biomass achieved about $60 \%$ of the theoretical carbohydrate conversion without refining, mechanical refining on the same biomass and with the same enzymatic hydrolysis conditions improved the conversion by an additional $15 \%$, resulting in a $75 \%$ carbohydrate conversion for the refined biomass. It is noted that refining reactivity also decreased below a certain point (60\% in Figure 2) by biomass recalcitrance, indicating some level of pretreatment is necessary to achieve maximum refining benefit. Based on the dotted line in Figure 2, one might consider producing pretreated biomass that is digested at $70 \%$ carbohydrate conversion under a certain set of enzymatic hydrolysis conditions (green arrow), so that the mechanical refining can enhance the overall carbohydrate conversion over $80 \%$. 
This plot clearly demonstrates the refining reactivity of a given substrate and can be used to investigate the correlations among various factors in pretreatment, mechanical treatment, and subsequent enzymatic hydrolysis. It is important to note that the maximum point and the width of the curve are likely highly dependent on the type of biomass, the pretreatment conditions, and the enzymatic hydrolysis conditions. Hence, to evaluate the effectiveness of mechanical refining for a specific biomass and a specific pretreatment condition, a refining reactivity study should be a prerequisite for further process development.

\subsection{Reduction in by-products formation}

Pretreatment of lignocellulosic biomass at more severe conditions (i.e. higher temperature, time, and chemical concentration) usually allows for greater carbohydrate conversions; however, this is often gained at the expense of lower pretreatment yield, higher process costs, and higher concentration of fermentation inhibitors (Pu et al., 2011; Pu et al., 2013). Lower pretreatment severities have been shown to generate fewer inhibitors such as acids, furans, and phenolic compounds (Klinke et al., 2004; Palmqvist and Hahn-Hägerdal, 2000a). The list of major inhibitors from the degradation of lignocellulosic biomass includes furfural, acetic acid, 5hydroxymethyl-2-furaldehyde (HMF), formic acid, and various phenolic compounds from the degradation of lignin. Some of these by-products can be toxic and inhibitory to biocatalysts and catalysts in fermentation and catalytic conversion and thus, it might be necessary to remove them prior to downstream process.

Figure 3 shows the difference in the amount of by-products from dilute-acid pretreated corn stover at different severities. Higher temperature and longer pretreatment time affected the composition and the amount of degradation products of lignocellulosic biomass. Increasing the temperature from 160 to $170^{\circ} \mathrm{C}$ and the residence time from 10 to $20 \mathrm{~min}$ significantly increased the amount of acetic acid and also generated furfural, which was not detected from the low severity pretreatment. Furthermore, a temperature increase from 160 to $190^{\circ} \mathrm{C}$ generated a substantial amount of furfural at the same pretreatment time $(10 \mathrm{~min})$. The effect of increased pretreatment severity on the degradation of xylan into furfural was reported (Kabel et al., 2007; Panagiotopoulos et al., 2011), and the inhibitory effect of increased pretreatment severity or elevated concentration of some by-products have been also investigated (Clark and Mackie, 1984; Laser et al., 2002; Palmqvist and Hahn-Hägerdal, 2000b). In addition, pretreatment with a lower severity will reduce the energy demand during the process, allow inexpensive reactor metallurgy, and provide flexibility in the process design and operation. In addition, it will generate fewer degradation products, resulting in notably lower inhibitory impacts on downstream biological conversion processes.

Therefore, to increase the overall carbohydrate conversion, it is necessary to find an optimal pretreatment severity, where larger amounts of hydrolysable carbohydrates and less inhibitory compounds for subsequent bioconversion processes can be attained effectively. In this context, the combination of chemical pretreatment followed by a mechanical refining posttreatment is considered to be a feasible option. 


\subsection{Energy consumption during mechanical refining}

With respect to the entire biorefinery process, refining is a relatively low energy demanding technology that can improve the carbohydrate conversion. In addition, its introduction to the biochemical conversion process will likely have little technological barriers to commercial application, as it is a well-established technology from the pulp and paper industry. Several studies have reported that there are close relationships between carbohydrate conversion and the applied energy during mechanical treatment. Zhu et al. presented that there was a positive correlation between the energy consumption and the glucose conversion. This elucidates the concept that the energy transfer to the biomass during mechanical refining increases the specific surface volume and opens up the biomass structure, which allows for more efficient cellulose conversion (Zhu et al., 2009b).

Although energy consumption might be estimated using a lab-scale mechanical treatment (PFI refiner), the actual value needs to be measured with a disc refining system. Recently, a pilot trial was performed with a 36-inch commercial scale disc refiner using alkali pretreated corn stover and the increased conversion was as shown in Figure 4 (Chen et al., 2014). The conversion was increased by $13 \%$ for glucose and $21 \%$ for xylose, respectively, with the refining energy input of $212 \mathrm{kWh} / \mathrm{ODMT}$ (oven dry metric ton). Since this refining process has been done by a relatively small-scale 36-inch refiner, it is expected to have a reduced energy demand when more efficient and larger scale commercial refiner equipment is used. In addition, since the reported study used the pretreated biomass at a solid content of $45 \sim 50 \%(\mathrm{w} / \mathrm{w})$ during refining, energy consumption at lower solid content will be much lower than the reported study. It is noted that solid content is likely to be $10 \sim 15 \%$ depending on the pretreatment severity if mechanical refining is directly followed by dilute-acid pretreatment as a continuous process. Considering these factors, it is reasonable to assume that the refining energy consumption might be about 100 $\mathrm{kWh} /$ ODMT. With a few assumptions based on NREL design report (NREL, 2011) including a) $271 \mathrm{~L}$ of ethanol can be produced from one ODMT of biomass, b) $74 \%$ pretreatment solids yield, and c) a retail price of electricity of $\$ 0.068 / \mathrm{kWh}$, the operating cost of mechanical refining to produce ethanol is about $\$ 0.018 / \mathrm{L}$ of ethanol ( $\$ 0.070 /$ gallon of ethanol). This result is consistent with a similar estimation by Jones et al. for woody biomass (Jones et al., 2014).

\subsection{Process considerations}

To consider mechanical refining for biochemical conversion process, it is necessary to evaluate the refining reactivity, i.e. the improvements in enzymatic conversion due to refining. As demonstrated in Figure 2, refining reactivity is largely dependent on biomass type, pretreatment severity, enzyme dosage, and others. It is noted that techno-economic analysis needs to be performed to fully understand the refining benefits. For example, a similar carbohydrate conversion to a process without refining might be achieved with mechanical refining with a reduced pretreatment severity. Then the economic benefits may come from lesser 
amounts of inhibitors and less expensive reactor metallurgy required, rather than increased carbohydrate conversion.

The point of application of the refining step in the process is also important. The mechanical refining could be directly after the pretreatment reactor, after solid/liquid separation following pretreatment, or another positions. If the refining is performed just after pretreatment, one of the advantages is that refining can be carried-out in-line under high temperature and pressure, which is beneficial to reduce the energy consumption during refining (Muhić et al., 2010). Even after the blow tank receiving the pretreated biomass with an elevated temperature, it can unburden the energy load during refining process. The other process layout, i.e. refining after solid/liquid separation for pretreated biomass, can be considered if one needs to separate xylose from the pretreated substrate, which can produce more specialized products. The liquid fraction can be separated from the solids by a hydraulic press, and then the solid fraction can undergo a washing step followed by mechanical refining. If a washing step is implemented, the solids content at refining can be adjusted to minimize energy consumption.

Solids content during mechanical refining is another important process consideration. It is directly correlated with energy consumption, and can also affect the properties of the refined biomass, based on the pulp consistency and refining process (Olejnik, 2013). Manipulation of solid consistency at refining can be achieved by controlling process factors, which are implemented throughout the biorefinery process layout including solid/liquid separation, washing, and dilution operation. Low consistency refining can greatly reduce the energy consumption during refining, but will also decrease the efficiency of the overall process since it requires an additional step for thickening or product purification (e.g. distillation) during downstream processes. High consistency refining can work with higher productivity, but the energy consumption will be increased accordingly. It should also be noted that low consistency refining tends to have more fiber-bar interactions. This interaction can result in fiber cutting by compression forces with edges of plate bars, whereas high consistency refining exhibits characteristics of more fiber-fiber interactions which tends to develop more external fibrillation and internal delamination.

\section{Conclusions}

Mechanical refining, widely used in the pulp and paper industry, can be directly applied to biochemical conversion processes. By implementing this technology, biomass recalcitrance can be overcome and carbohydrate conversion can be enhanced with commercially attractive levels of enzymes. Pretreatment severity can also be reduced to achieve the same level of sugar recovery, which can reduce the pretreatment cost and result in lower concentrations of inhibitors. Refining is a versatile and commercially proven technology at process flows of up to $~ 1,500$ dry tons of biomass/day. With judicious application, mechanical refining can make an overall biorefinery process more economically viable. 


\section{Acknowledgement}

This study was jointly supported by US DOE National Renewable Energy Laboratory (Subcontract No. XEV-5-42083-01) and Korea Evaluation Institute of Industrial Technology (KEIT) Research Grant of 2014 (TS141-21R). 


\section{References}

1. Brändström, J., Joseleau, J.-P., Cochaux, A., Giraud-Telme, N., Ruel, K., 2005. Ultrastructure of commercial recycled pulp fibers for the production of packaging paper. Holzforschung, 59(6), 675-680.

2. Chen, H., Venditti, R.A., Jameel, H., Park, S., 2012a. Enzymatic hydrolysis of recovered office printing paper with low enzyme dosages to produce fermentable sugars. Appl. Biochem. Biotechnol., 166(5), 1121-1136.

3. Chen, X., Kuhn, E., Wang, W., Park, S., Flanegan, K., Trass, O., Tenlep, L., Tao, L., Tucker, M., 2013. Comparison of different mechanical refining technologies on the enzymatic digestibility of low severity acid pretreated corn stover. Bioresour. Technol., 147, 401-408.

4. Chen, X., Shekiro, J., Pschorn, T., Sabourin, M., Tao, L., Elander, R., Park, S., Trass, O., Flanegan, K., Nelson, E., Jennings, E., Nelson, R., Johnson, E., Tucker, M.P., 2014. A novel transformational process replacing dilute acid pretreatment with deacetylation and mechanical (disc) refining for the conversion of renewable biomass to lower cost sugars. 36th Symposium on Biotechnology for Fuels and Chemicals, Clearwater Beach, FL.

5. Chen, X., Tao, L., Shekiro, J., Mohaghaghi, A., Decker, S., Wang, W., Smith, H., Park, S., Himmel, M.E., Tucker, M., 2012b. Improved ethanol yield and reduced Minimum Ethanol Selling Price (MESP) by modifying low severity dilute acid pretreatment with deacetylation and mechanical refining: 1) Experimental. Biotechnol. Biofuels, 5(60), 110.

6. Clark, T.A., Mackie, K.L., 1984. Fermentation Inhibitors in Wood Hydrolysates Derived from the Softwood Pinus-Radiata. J. Chem. Tech. Biot. B, 34(2), 101-110.

7. Ertas, M., Han, Q., Jameel, H., Chang, H.-M., 2014. Enzymatic hydrolysis of autohydrolyzed wheat straw followed by refining to produce fermentable sugars. Bioresour. Technol., 152, 259-266.

8. Franco, H., Ferraz, A., Milagres, A.M.F., Carvalho, W., Freer, J., Baeza, J., Mendonça, R.T., 2012. Alkaline sulfite/anthraquinone pretreatment followed by disk refining of Pinus radiata and Pinus caribaea wood chips for biochemical ethanol production. J. Chem. Technol. Biotechnol., 87(5), 651-657.

9. Gil, N., Gil, C., Amaral, M.E., Costa, A.P., Duarte, A.P., 2009. Use of enzymes to improve the refining of a bleached Eucalyptus globulus kraft pulp. Biochem. Eng. J., 46(2), 89-95.

10. Han, Q., Jin, Y.B., Jameel, H., Chang, H.-M., Phillips, R., Park, S., 2015. Autohydrolysis pretreatment of waste wheat straw for cellulosic ethanol production in a co-located straw pulp mill. Appl. Biochem. Biotech., 175(2), 1193-1210.

11. Himmel, M.E., Ding, S.Y., Johnson, D.K., Adney, W.S., Nimlos, M.R., Brady, J.W., Foust, T.D., 2007. Biomass recalcitrance: Engineering plants and enzymes for biofuels production. Science, 315(5813), 804-807. 
12. Hoeger, I.C., Nair, S.S., Ragauskas, A.J., Deng, Y., Rojas, O.J., Zhu, J.Y., 2013. Mechanical deconstruction of lignocellulose cell walls and their enzymatic saccharification. Cellulose, 20(2), 807-818.

13. Høydahl, H.E., Solbakken, M., Dahlqvist, G., 1995. TMP for SC-grades - A challenge in fiber modelling. International Mechanical Pulping Conference Ottawa, Canada. 233-237.

14. Huo, D., Fang, G., Yang, Q., Han, S., Deng, Y., Shen, K., Lin, Y., 2013. Enhancement of eucalypt chips' enzymolysis efficiency by a combination method of alkali impregnation and refining pretreatment. Bioresour. Technol., 150, 73-78.

15. Jones, B.W., Venditti, R., Park, S., Jameel, H., 2014. Comparison of lab, pilot, and industrial scale low consistency mechanical refining for improvements in enzymatic digestibility of pretreated hardwood. Bioresour. Technol., 167(0), 514-520.

16. Jones, B.W., Venditti, R., Park, S., Jameel, H., Koo, B., 2013. Enhancement in enzymatic hydrolysis by mechanical refining for pretreated hardwood lignocellulosics. Bioresour. Technol., 147, 353-360.

17. Ju, X., Grego, C., Zhang, X., 2013. Specific effects of fiber size and fiber swelling on biomass substrate surface area and enzymatic digestibility. Bioresour. Technol., 144, 232-239.

18. Kabel, M.A., Bos, G., Zeevalking, J., Voragen, A.G.J., Schols, H.A., 2007. Effect of pretreatment severity on xylan solubility and enzymatic breakdown of the remaining cellulose from wheat straw. Bioresour. Technol., 98(10), 2034-2042.

19. Kerekes, R.J., 1990. Characterization of pulp refiners by a C-factor. Nord. Pulp Pap. Res. J., 5(1), 3-8.

20. Kibblewhite, R.P., 1972. Effect of beating on fibre morphology and fibre surface structure. Appita, 26(3), 196-202.

21. Klinke, H.B., Thomsen, A.B., Ahring, B.K., 2004. Inhibition of ethanol-producing yeast and bacteria by degradation products produced during pre-treatment of biomass. Appl. Microbiol. Biot., 66(1), 10-26.

22. Koo, B.W., Treasure, T.H., Jameel, H., Phillips, R.B., Chang, H.-M., Park, S., 2011. Reduction of enzyme dosage by oxygen delignification and mechanical refining for enzymatic hydrolysis of green liquor-pretreated hardwood. Appl. Biochem. Biotechnol., 165(3-4), 832-844.

23. Kure, K.A., Dahlqvist, G., 1998. Development of structural fibre properties in high intensity refining. Pulp \& paper Canada, 99(7), 59-63.

24. Kure, K.A., Dahlqvist, G., Sabourin, M.J., Helle, T., 1999. Development of spruce fibre properties by a combination of a pressurized compressive pretreatment and high intensity refining. International Mechanical Pulping Conference, Houston, US. 427-433.

25. Kure, K.A., Sabourin, M.J., Dahlqvist, G., Helle, T., 2000. Adjusting refining intensity by changing refiner plate design and rotational speed - Effects on structural fiber properties. J. Pulp Paper Sci., 26(10), 346-352. 
26. Laser, M., Schulman, D., Allen, S.G., Lichwa, J., Antal, M.J., Lynd, L.R., 2002. A comparison of liquid hot water and steam pretreatments of sugar cane bagasse for bioconversion to ethanol. Bioresour. Technol., 81(1), 33-44.

27. Miles, K.B., 1991. A simplified method for calculating the residence time and refining intensity in a chip refiner. Paperi ja Puu, 73(9), 852-857.

28. Molin, U., Daniel, G., 2004. Effects of refining on the fibre structure of kraft pulps as revealed by FE-SEM and TEM: Influence of alkaline degradation. Holzforschung, 58(3), 226-232.

29. Molin, U., Lennholm, H., 2000. The influence of molar mass on mechanical properties of pulp fibers. 54 ${ }^{\text {th }}$ Appita Annual Conferece, Melbourne, Australia. Appita. 615-621.

30. Muhić, D., Sundström, L., Sandberg, C., Ullmar, M., Engstrand, P., 2010. Influence of temperature on energy efficiency in double disc chip refining. Nord. Pulp Pap. Res. J., 25, 420-427.

31. NREL, 2011. Process design and economics for biochemical conversion of lignocellulosic biomass to ethanol. National Renewable Energy Laboratory. NREL/TP5100-47764.

32. Olejnik, K., 2013. Impact of pulp consistency on refining process conducted under constant intensity determined by SEL and SEC factor. Bioresources, 8(3), 3212-3230.

33. Page, D.H., 1989. The beating of chemical pulps - The action and the effects. in: Transactions of the Ninth Fundamental Research Symposium. Cambridge, UK, pp. 1-37.

34. Palmqvist, E., Hahn-Hägerdal, B., 2000a. Fermentation of lignocellulosic hydrolysates. I: inhibition and detoxification. Bioresour. Technol., 74(1), 17-24.

35. Palmqvist, E., Hahn-Hägerdal, B., 2000b. Fermentation of lignocellulosic hydrolysates. II: inhibitors and mechanisms of inhibition. Bioresour. Technol., 74(1), 25-33.

36. Panagiotopoulos, I.A., Bakker, R.R., de Vrije, T., Koukios, E.G., 2011. Effect of pretreatment severity on the conversion of barley straw to fermentable substrates and the release of inhibitory compounds. Bioresour. Technol., 102(24), 11204-11211.

37. Park, S., Venditti, R.A., Jameel, H., Pawlak, J.J., 2006. Changes in pore size distribution during the drying of cellulose fibers as measured by differential scanning calorimetry. Carbohyd. Polym., 66(1), 97-103.

38. Pu, Y., Treasure, T., Gonzalez, R., Venditti, R., Jameel, H., 2011. Autohydrolysis pretreatment of mixed hardwoods to extract value prior to combustion. Bioresources, 6(4), 4856-4870.

39. Pu, Y., Treasure, T., Gonzalez, R., Venditti, R.A., Jameel, H., 2013. Autohydrolysis pretreatment of mixed softwood to produce value prior to combustion. Bioenerg. Res., 6(3), 1094-1103.

40. Sabourin, M., Xu, E., Musselman, R., 1997. Evaluation of refiner disc speed, plate design and consistency on high pressure/low residence (RTS) TMP pulps. International Mechanical Pulping Conference, Stockholm, Sweden. 241-249. 
41. Smook, G.A., 2002. Handbook for Pulp \& Paper Technologists. 3rd ed. Angus Wilde Publications.

42. Stoere, P., Nazhad, M., Kerekes, R., 2001. An experimental study of the effect of refining on paper formation. Tappi, 84(7), 52.

43. Szwarcsztajn, E., Przybysz, K., 1972. External fibrillation of beaten cellulose fibers. Cellul. Chem. Technol., 6(2), 223-238.

44. Teder, A., 1964. The properties of spruce pulps as related to paper structures observed with the scanning electron microscope. Svensk Papperstidn, 67(17), 670-685.

45. Wu, S., Chang, H.-M., Jameel, H., Philips, R., 2010. Novel green liquor pretreatment of loblolly pine chips to facilitate enzymatic hydrolysis into fermentable sugars for ethanol production. J. Wood Chem. Technol., 30(3), 205-218.

46. Xu, H.F., Li, B., Mu, X.D., Yu, G., Liu, C., Zhang, Y.D., Wang, H.S., 2014. Quantitative characterization of the impact of pulp refining on enzymatic saccharification of the alkaline pretreated corn stover. Bioresour. Technol., 169, 19-26.

47. Zhu, J.Y., Pan, X.J., Wang, G.S., Gleisner, R., 2009a. Sulfite pretreatment (SPORL) for robust enzymatic saccharification of spruce and red pine. Bioresour. Technol., 100(8), 2411-2418.

48. Zhu, J.Y., Wang, G.S., Pan, X.J., Gleisner, R., 2009b. Specific surface to evaluate the efficiencies of milling and pretreatment of wood for enzymatic saccharification. Chem. Eng. Sci., 64(3), 474-485.

49. Zhu, W., Zhu, J.Y., Gleisner, R., Pan, X.J., 2010. On energy consumption for sizereduction and yields from subsequent enzymatic saccharification of pretreated lodgepole pine. Bioresour. Technol., 101(8), 2782-2792. 


\section{Figure captions}

Figure 1. The effect of mechanical refining on the enzymatic hydrolysis of pretreated lignocellulosic biomass. a) With $2 \mathrm{k}$ revolutions in a PFI refiner using dilute-acid pretreated corn stover, the same level of conversion (78\%) was achieved with half the dosage of enzyme, compared to unrefined pretreated biomass. No difference was observed over $4 \mathrm{k}$ refining (82\%), indicating enzyme savings at moderate refining severity. b) Improvement in carbohydrate conversion by mechanical refining with different severity on green-liquor pretreated loblolly pine. Compared to unrefined samples (0k), PFI-refining with up to $8 \mathrm{k}$ revolutions clearly increased the carbohydrate conversion.

Figure 2. Absolute hydrolysis improvement (y-axis) due to the refining (PFI 4k revolutions) on unrefined alkaline-pretreated biomass (x-axis). The dotted line shown is the best fit for visualization purposes. The slope and maximum of the curve are subject to change with biomass type, pretreatment chemistry, and enzyme hydrolysis conditions. The circled area is the target unrefined carbohydrate conversion range to achieve maximum overall carbohydrate conversion from the process.

Figure 3. The amount of by-product inhibitors during dilute acid pretreatment of corn stover with different pretreatment temperature and time; CS160-10 refers the dilute acid pretreated corn stover sample at $160^{\circ} \mathrm{C}$ for $10 \mathrm{~min}$.

Figure 4. Yield of monomeric glucose and xylose from low solids enzymatic hydrolysis of deacetylated corn stover with and without disc-refining, as a function of energy consumption during refining. It was observed that close to 20 percent points increase in both glucose and xylose conversion yield can be achieved with relatively low energy consumption by mechanical refining (Chen et al., 2014). 
Table 1. Specific energy input for the major pulp grades.

\begin{tabular}{|c|c|c|c|}
\hline \multirow{2}{*}{ Grade } & Pulp & $\begin{array}{c}\text { Net specific } \\
\text { energy (kWh/t) }\end{array}$ & $\begin{array}{c}\text { Refining intensity } \\
\text { (Ws/m) }\end{array}$ \\
\hline \multirow{2}{*}{ Fine Paper } & Hardwood & $30-120$ & $0.3-0.8$ \\
\cline { 2 - 4 } & Softwood & $40-120$ & $2.0-4.0$ \\
\hline \multirow{2}{*}{ Newspaper } & Softwood Kraft & $20-60$ & $1.0-2.5$ \\
\cline { 2 - 4 } & Groundwood & $20-60$ & $0.2-0.5$ \\
\hline Linerboard & OCC & $30-60$ & $0.2-0.8$ \\
\hline
\end{tabular}


Figure 1.
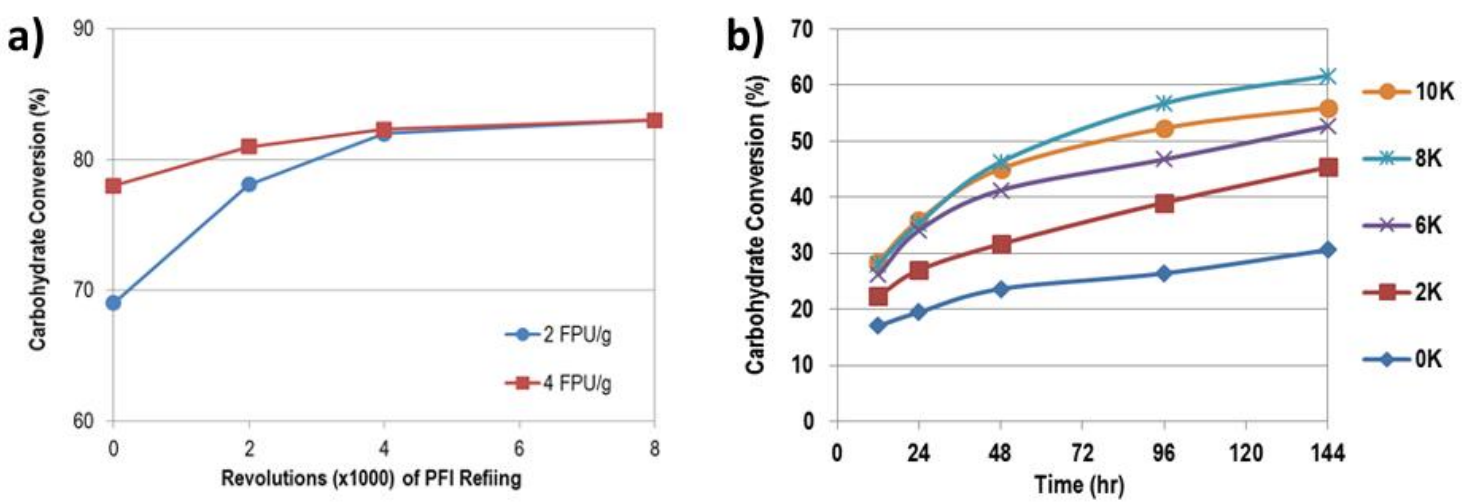
Figure 2.

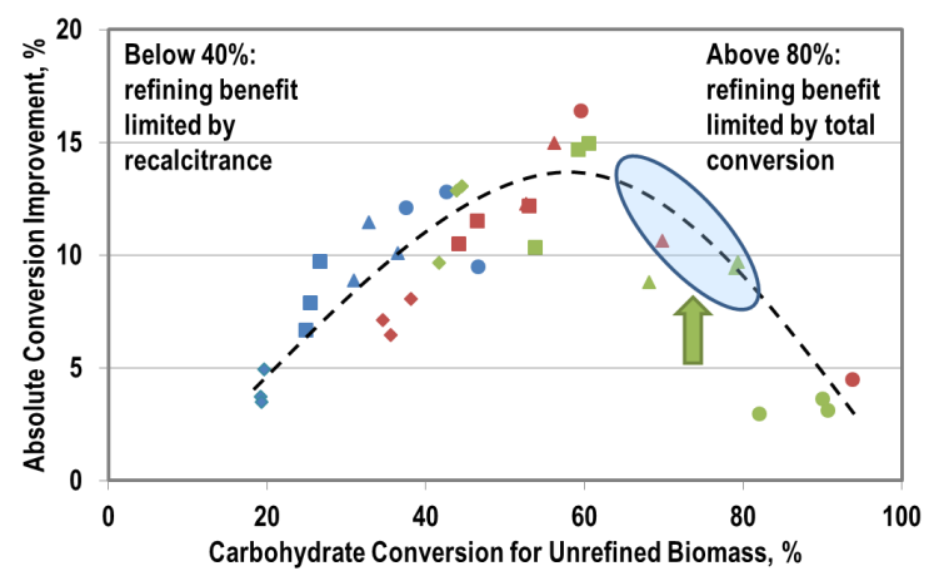


Figure 3.

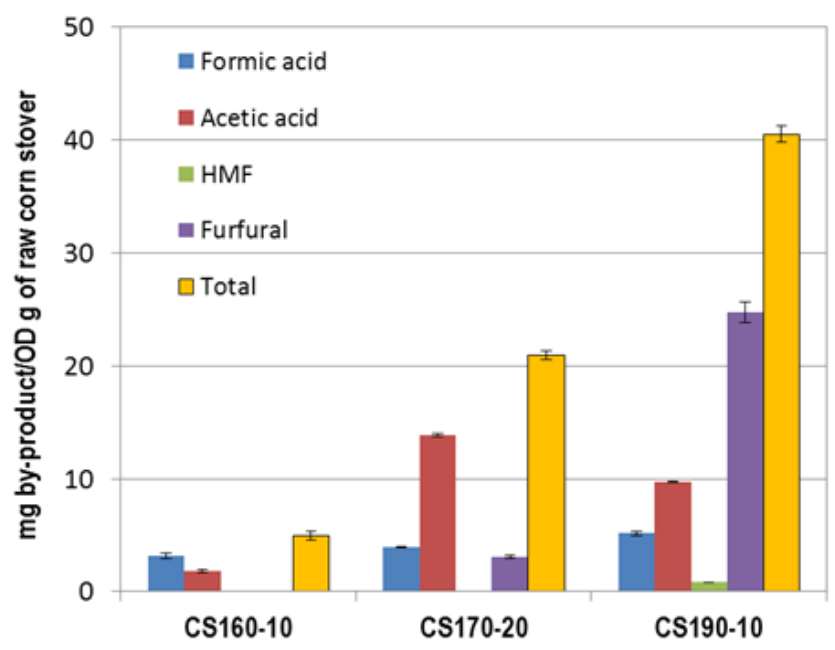


Figure 4.

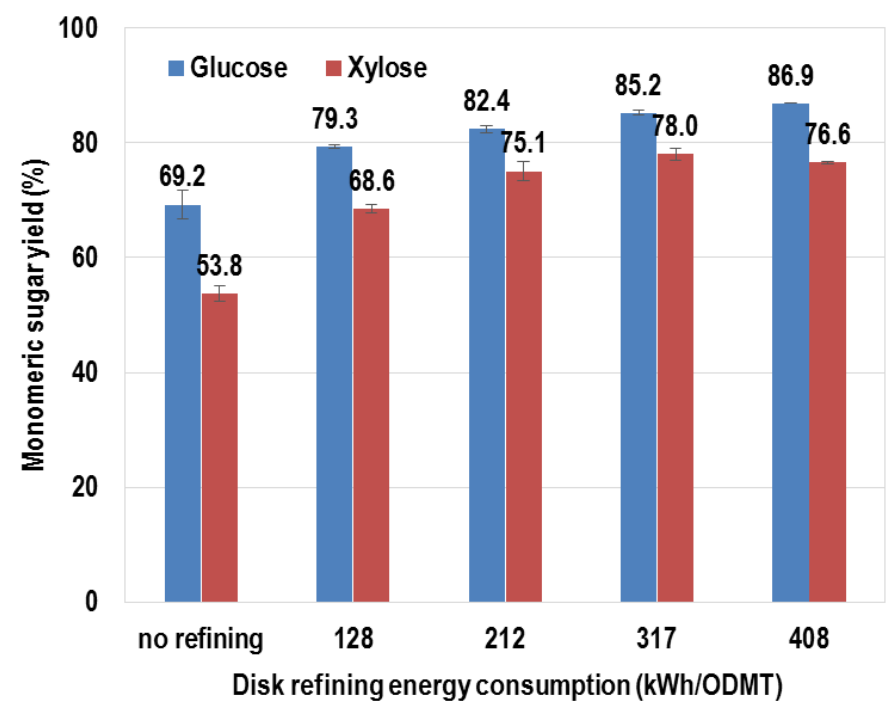

